ISSN 1112-9867

\title{
METABOLIC COST DURING TYRE AND ROPE FUNCTIONAL TRAINING
}

\author{
M. M. Iskandar ${ }^{1 *}$, N. I. Mohamad ${ }^{1}$, S. Othman ${ }^{2}$, and A. M. Nadzalan ${ }^{1}$ \\ ${ }^{1}$ Faculty of Sports Science and Coaching, Universiti Pendidikan Sultan Idris, Tanjung Malim, \\ Perak, Malaysia \\ ${ }^{2}$ Faculty of Science and Mathematics, Universiti Pendidikan Sultan Idris, Tanjung Malim, \\ Perak, Malaysia.
}

Published online: 10 November 2017

\begin{abstract}
This study aimed to examine the metabolic cost during tyre and rope functional training. 20 male youth aged between 20 and 24 years old with average age $(22.05 \pm 1.46)$ were recruited for this study. Participants involved in three types of exercise that uses rope and three types of exercise using a tire. Metabolic cost (Metabolic Equivalent of Task value, oxygen consumption, energy cost, and pulse rate training) during training was measured using a special tool called portable metabolic analyzer. Results showed the average MET value for exercises using rope and tires for 10 minutes was $7.25 \pm 1.62$. The average recorded oxygen consumption was $1655.05 \pm 295.703) \mathrm{mL} \cdot \min -1$. While the average energy used is $74.75 \pm$ $14.78 \mathrm{kcal}$ or $7.47 \mathrm{kcal} \cdot \min -1$. The average pulse rate during a 10 -minute exercise function was $153.15 \pm 13.28$ per minute. Overall, using rope and tire as tools for resistance training would give many advantages for the functionality development among the individuals.
\end{abstract}

Keywords: metabolic cost; rope; tyre; oxygen; functionality.

Author Correspondence, e-mail: mohni_iskandar@yahoo.com doi: http://dx.doi.org/10.4314/jfas.v9i6s.77 


\section{INTRODUCTION}

Training to improve body functional motion using functional based exercises has become increasingly popular. Movement which performed using various types of equipment or body weight, which targeted various muscles crossing multiple joints, and involving more than one movement plane has been considered as 'functional exercise' [1, 2]. Meanwhile, Cosio-Lima et al. [3] describe functional training as the ability of the neuromuscular system to stabilize the body through dynamic and isometric contractions in response to stressors such as gravity, ground reaction forces and momentum.

For training to be functional, exercise training program should be designed to mimic tasks or activities that occur in a person's daily life to make training adaptations more transferable [4-6] and variables must be controlled and monitored, so that the prescription has the proper dose of exercise that the individual must perform in the training unit [7]. The idea and concept of functional training promote the notion that these training are replicates natural daily movement and included movement-based exercise to improve person's ability to achieve a specific goal, thus avoiding focusing solely on specific muscular adaptations as in traditional exercise programs. Traditional exercise programs are commonly thought to involve exercises that isolate specific muscles in order to increase strength more effectively [8].

According to Fleck \& Kraemer [4] exercises using various objects as resistance to lift are known as resistance exercises and among the commonly used objects in this exercise are kettlebells, logs, sandbags, tires and hammer [9-11]. Large tires can produce a great barrier in exercise. For example, the tire flipping action involves the extension of three parts of the body namely the waist, knee, and ankle which is effective in improving the strength and power [9, 12]. Tire flips are also suitable for an anaerobic physical exercise training as this exercise can increase pulse rate and blood lactate $[12,13]$.

In addition to tires, Battle Rope can also be used as resistance. Rope exercise has become a popular fitness activity at the fitness centers and physical experts also use rope workouts in their training program [14]. A study conducted by Climstein \& Walsh [15], found that Battling Rope's exercise improved the use of oxygen, energy consumption, and increased pulse rate higher than traditional-style resistance exercises such as bench press, squat, and 
deadlift. Fountaine \& Schmidt [16] states that Battling Rope exercises are high-intensity exercises and potentially increasing cardiovascular fitness.

\section{METHODOLOGY}

\subsection{Participants}

The participants of the study involved 20 male youth aged between 20 and 24 years old with average age $(22.05 \pm 1.46)$. The participants who were recruited in this study consisted of students from the Universiti Pendidikan Sultan Idris who were voluntarily involved in this study. The average body mass and height of the participants was $68.61 \pm 2.66 \mathrm{~kg}$ and $1.68 \pm$ $0.23 \mathrm{~m}$, while the average body mass index was $24.16 \pm 1.82 \mathrm{~kg} / \mathrm{m}^{2}$. Before the survey, participants were informed in detail about the goals and protocols of the study that will be carried out. Written consent is obtained from each participant and they are also informed that they are free to withdraw from the study at any time To ensure that the participants are fit and free from health problems, all participants have undergone a screening test of readiness to do physical activity using the Physical Activity Readiness Questionnaire (PAR-Q) which was built by the Canadian Sociaty of Exercise Physiology. This study has also been approved by the Universiti Pendidikan Sultan Idris.

\subsection{Exercise protocols}

This study aimed to produce descriptive data about the metabolic cost generated during an exercise session using tires and rope. This exercise program consists of three types of exercise that uses rope and three types of exercise using a tire (Table 1). The straps used in this exercise are the 17.4-meter manila strap, $3.8 \mathrm{~cm}$ in diameter, and weights $17.5 \mathrm{~kg}$. The 17.4-meter rope is folded into two (8.7 meters each) and the center of the rope is fastened to the ballast so that it will not move. The tires used in this exercise are used tires from heavy machinery (tractors) weighing $74.2 \mathrm{Kg}$. Each participant is required to perform a 10-minute exercise session with the same prescription (Table 1). Each exercise is done within 30 seconds with maximum repeatability. The maximum repeat means the participant performs a number of repetitions within the given time frame. After performing the first exercise, the participants relax for 60 seconds before doing the second exercise and the next exercise up to 6 complete 
exercises with the same rest period between exercises. The duration of exercise and the rest of the exercise are monitored by the appointed testers. In this study, metabolic cost (Metabolic Equivalent of Task value, oxygen consumption, energy cost, and pulse rate training) during training was measured using a special tool called portable metabolic analyzer (Fitmate pro, Cosmed, Italy). The validity of this tool has been reported ie $r=0.97$ [17]. The body weight of the participants was measured using the weighing scale (HN-283, Omron, Kyoto, Japan) while height of the participants were measured using (SEC213, Seca, USA).

Table 1. Tyre and rope exercises performed in this study

Exercises
At standing position, the knees are slightly bent and the legs are in the open position
(shoulder width). Hold both ends of the rope firmly, and place your hands in front of the
waist. Strengthen the core muscles that are the spine and abdomen of the muscles, then move
both hands up and down rapidly to produce a waveform on the rope.

\section{Tire Flip}

Stand against the tires laid horizontally on the floor. Bend the knee (squat) and place both hands under the tire. Contract the core muscles and 'lock' the waist, then pull and lift the tire up using both hands while pushing the legs down. Then push the tire forward until the tire is fully flip. Repeat the movement several times 

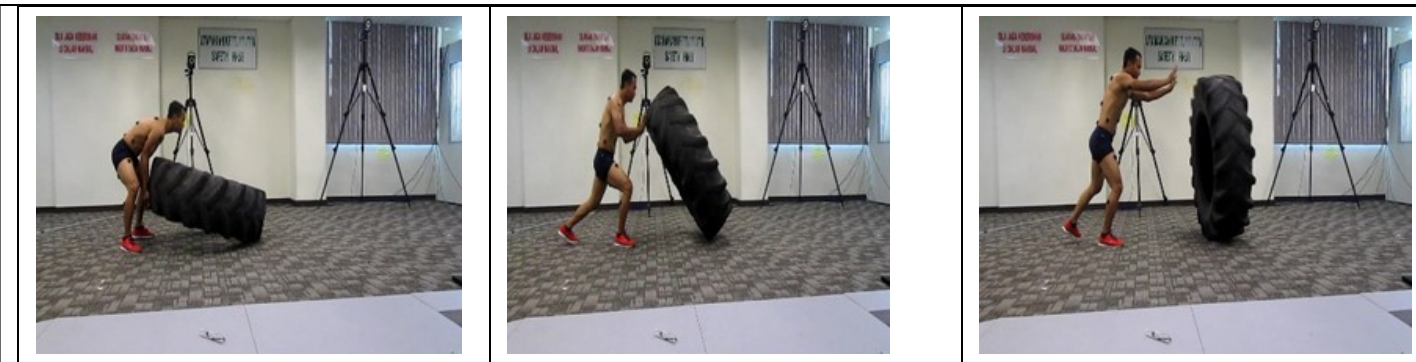

\section{Clockwise}

At a standing position, the knee is slightly bent and the legs are in shoulder with opend. Hold both ends of the rope firmly, and place your hands in front of the waist. Contracts the core muscles and rotate the shoulder with the right arm rotating clockwise and the left arm rotates in opposite directions. Repeat this movement quickly and continuously.
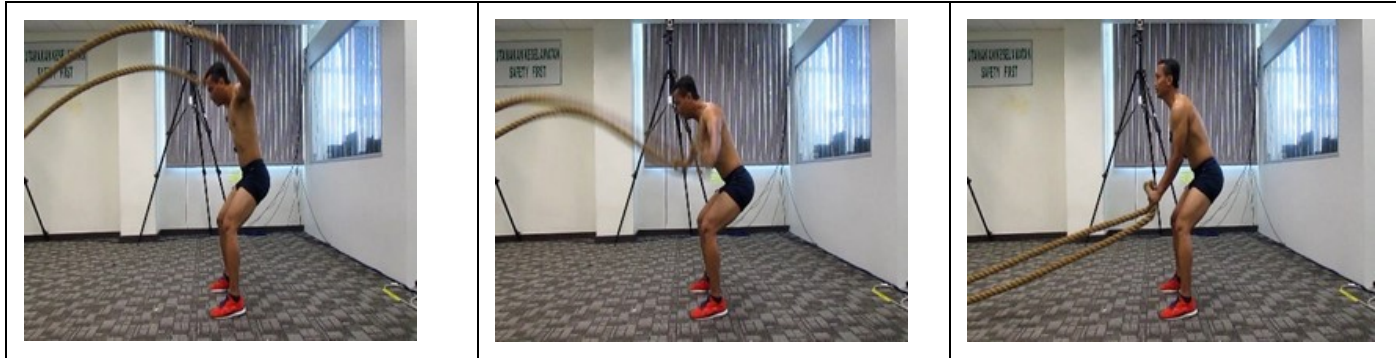

\section{Bent Over Row}

Stand inside the center of the tire circle with a slightly bent knee and foot open (shoulder width). Bend the waist forward and hold the inside part of the tire. The back, neck, and head need to be parallel. Contracts the core muscles, lock the waist, and knees slightly bent. Then push the tire up using the foot by pushing the foot down and straightening the knees and arms should always be straight. Next, bend your knees and lower the tire down slowly to the original position. Repeat the movement several times.

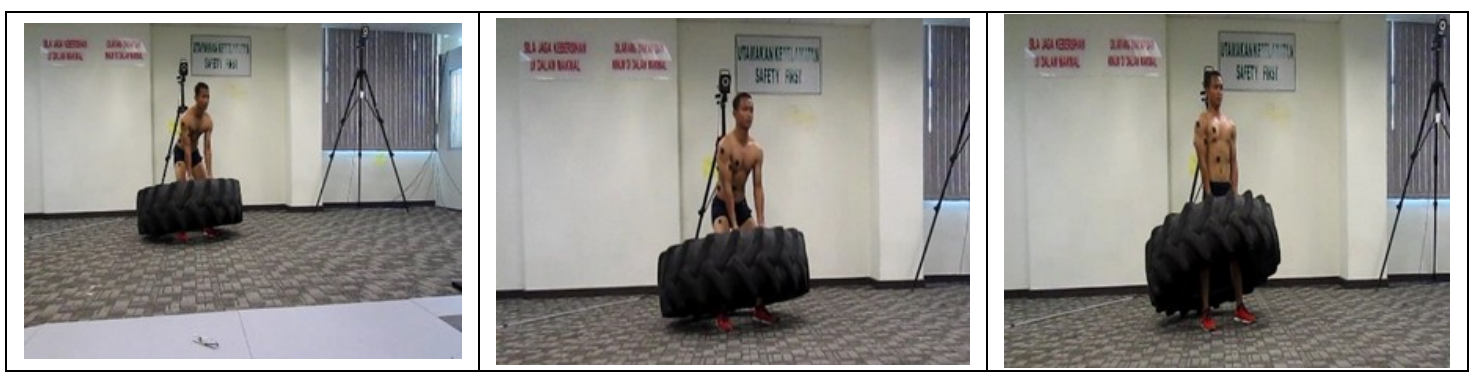




\section{Hip Toss}

At standing position, the knees are slightly bent and the legs are in the open position (shoulder width). Hold both ends of the string firmly, and place both hands on the right side of the body at the waist level. Contract the core muscles and lock the waist while swinging the hand that holds the rope to the left and right side of the body repeatedly.
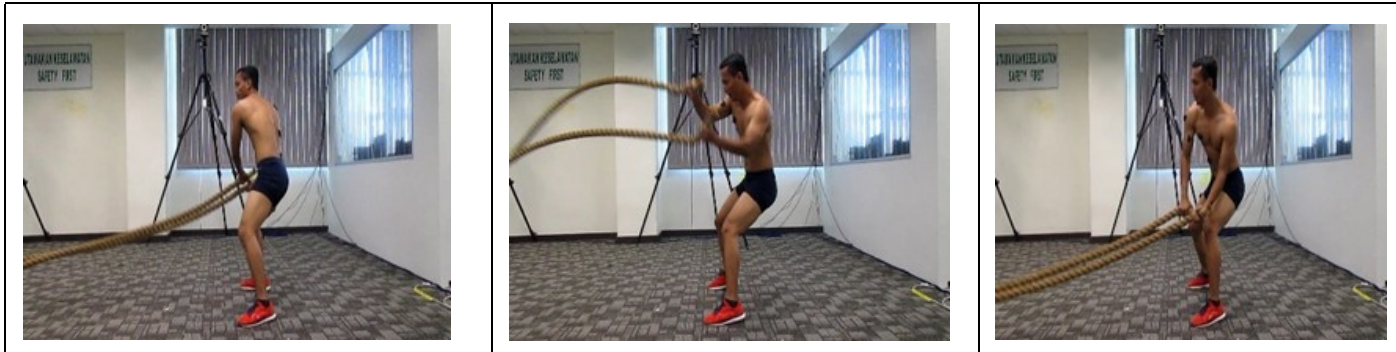

\section{Sledgehammer}

Stand in front of the tire with a slightly bent knee. Hold the iron hammer with both hands and place it on the tire. Contracts the core muscles and lock the waist, then lift the iron hammer and carry the back of the body with the arms behind the head, and then push the iron hammer forward and hit the tire firmly. Repeat the movement several times.
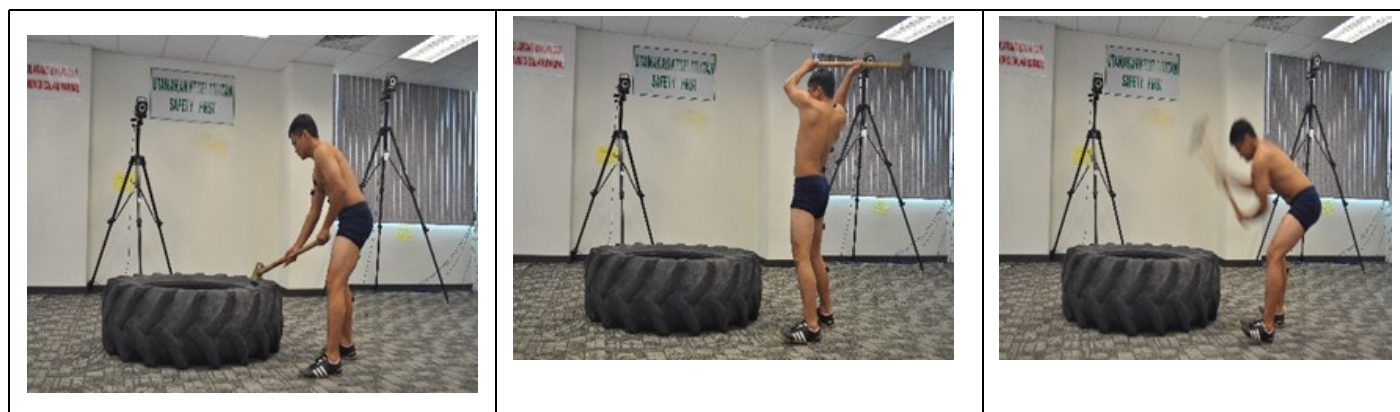
Table 2. Exercise program prescription for measurement of metabolic cost

\begin{tabular}{|l|l|l|c|}
\hline Senaman & Repetitions & Set & Exercise Duration (s) \\
\hline Double Waves (rope) & maximum & 1 & 30 \\
\hline \multicolumn{3}{|c|}{ Rest } & 60 \\
\hline Tyre Flip (tire) & maximum & 1 & 30 \\
\hline \multicolumn{3}{|c|}{ Rest } & 60 \\
\hline Clockwise (tire) & maximum & 1 & 30 \\
\hline \multicolumn{3}{|l|}{ Rest } & 60 \\
\hline Squat (tire) & maximum & 1 & 30 \\
\hline \multicolumn{2}{|l|}{ Rest } & 60 \\
\hline Hip Toss (rope) & maximum & 1 & 30 \\
\hline & Rest & 60 \\
\hline Sledgehammer (tire) & maximum & 1 & 30 \\
\hline
\end{tabular}

\subsection{Procedures}

Before the study, each participant attended the briefing session and demonstrated how the exercise was conducted. At this session, the height and body mass of each participant was recorded. Next, participants were asked to try and practice each exercise they had shown. The movement of the participants is assessed and corrected if the movements made by the participants are not accurate. This briefing session and demonstration are conducted in the same place for each participant. Participants are also reminded not to take any heavy physical activity within three days before the test is conducted.

On the day of testing, participants were asked to do warm-up activities and to try each exercise. The weight and body mass data of the participants are recorded and included in the metabolic analyzer. Once ready, the researcher is subjected to a respiratory mask connected to a portable metabolic analyzer placed in a backpack bearing on the back of the body. This device is compact $(20 \times 24 \mathrm{~cm})$, portable, lightweight, and does not preclude any movement by the subject. Once the mobile analyzer metabolic is activated, the participants are given a signal to perform the exercise according to the prescription and predefined protocols. During 
testing, participants are asked to breathe normally and are not allowed to speak. Start and stop instructions for each exercise and rest are given to participants by a research assistant (certified fitness trainer). Once the test is terminated, the metabolic cost data shown on the display screen is printed and recorded. Testing was carried out at the same place, at the physical laboratory, Faculty of Sports Science and Coaching, UPSI, with room temperature adjusted at $27^{\circ} \mathrm{C}$.

\subsection{Statistical Analysis}

The raw data for the tested variables, the Metabolic Equivalent of Task, the use of oxygen, energy costs, and the pulse rate of the training measured using the metabolic analyzer was calculated automatically. Descriptive analysis of mean and standard deviation was done using Statistical Packages for Social Science for Window 20.0 (SPSS). Normality tests have also been conducted to ensure that the data obtained is normal.

\section{RESULTS}

Table 3. Metabolic profile for battle-rope and tire flip exercise for 10 minutes.

MET

$\mathrm{VO}_{2}$

Energy Cost (Kcal)

Average HR

$$
7.25 \pm 1.62
$$

$1655.05 \pm 295.70$

$74.75 \pm 14.78$

$153.15 \pm 13.28$

The results of the descriptive analysis are shown in Table 3. All the data presented are mean and standard deviation. The findings from this study show that the average MET value for exercise exercises using rope and tires for 10 minutes is $7.25 \pm 1.62$. The average recorded oxygen consumption was $1655.05 \pm 295.703) \mathrm{mL} \cdot \mathrm{min}^{-1}$. While the average energy used is $74.75 \pm 14.78 \mathrm{kcal}$ or $7.47 \mathrm{kcal} \cdot \mathrm{min}^{-1}$. While the average pulse rate during a 10 -minute exercise function was $153.15 \pm 13.28$ per minute. 


\section{DISCUSSIONS}

The study aims to produce metabolic cost profile for exercise using tires and rope. The study also aims to determine whether exercise using the tires and the rope can achieve the intensity of exercise recommended by ACSM [18] to remain active. Based on the recorded MET value of $7.25 \pm 1.62$, this training can be categorized as high intensity training because according to Ainsworth et al. [19], physical activity having MET value $\geq 6$ is a vigorous physical activity. The value of MET 6 is often used as a weight to heavy physical activity because this value usually corresponds to a maximum capacity of $60 \%$ for most people, but once this value will be very low for young or active people and to be very high for the elderly or not fit [20].

1 MET represents the average cost of an adult's break-up energy that is determined by the amount of 1 calorie per kilogram of body weight for 1 hour $\left(1 \mathrm{kcal} \cdot \mathrm{kg}^{-1} \cdot \mathrm{hr}^{-1}\right)$ or representing the amount of oxygen consumption 3.5 milliliters per kilogram weight for 1 minute $(3.5 \mathrm{mLkg}$ ${ }^{-1} \cdot \min ^{-1}$ ) [21-23]. Based on the compendium (summary) of physical activity $[19,24]$, the MET value of this exercise is 7.3 higher than some recreational sports activities such as ball games volleyball 6.0 MET, 5.5 MET badminton, 6.0 MET basketball, 7.0 MET football, and also higher than 6 MET load training.

In addition to the MET value, training intensity can also be determined or expressed as a percentage of the maximum pulse rate. Based on this method, the intensity of the function training using the tires and the strings is determined by means of: training pulse rate (153.15) / 220 - age (22.05) x $100=77.34 \%$. Based on guidelines issued by ACSM [18], exercises that increase pulse rate between 77 to $95 \%$ of maximum pulse rate are exercises categorized as high-intensity exercises and able to enhance cardiovascular fitness.

Based on the search on some database, researchers found that no research has resulted in a metabolic cost profile of training that combines tires and ropes in one training session. To make a direct comparison with past studies is difficult because there is a clear distinction between variables such as population, training protocol, and also the type of resistance training used. Among the recent studies that closely resembled this study were the study conducted by Fountaine \& Schmidt [16], which measures the metabolic cost for a batting exercise (Battling Rope) for 10 minutes. Exercise is done with a 15-minute interval and a break for 45 seconds. Results from the study showed that this exercise can increase the heart 
rate of $163 \pm 11$ per minute, ie reaching a training intensity of about $86 \%$ based on the maximum rate of pulse rate. The energy consumption rate is $467.3 \pm 161.0 \mathrm{~kJ}$, while the recorded MET value is $10.1 \pm 1.6 \mathrm{~mL} . \mathrm{kg}^{-1} . \mathrm{min}^{-1}$.

Climstein \&. Walsh [15] also conducted a study on metabolic costs for Battling Rope exercises, finding out that the exercise performed with a 30 -minute interval with maximum repeat counts and rest pauses for 2 minutes can increase the pulse rate of 153 per minute, reaching training intensity of about $77 \%$ of the maximum pulse rate. Whereas oxygen consumption reaches $24.6 \mathrm{~mL} . \mathrm{kg}^{-1} \cdot \mathrm{min}^{-1}$, and the recorded energy consumption rate is 10.3 $\mathrm{kcal} \cdot \min ^{-1}$. Based on these two previous studies, it was found that the training pulse rate and energy consumption rates for exercise exercises using tires and strings were lower than training using only Battling rope in a training session. This may be due to the heavy use of tires in training in preventing participants from performing fast and much more than Battling Rope exercises. This is because the number or frequency of repetition done in each exercise set can affect the energy consumption rate [25].

However, the pulse rate and energy costs resulting from the training of this function are higher if compared with some types of traditional resistance training conducted by previous researchers. Metabolic cost of free-use circuit training by Beckham, \& Earnest [26], showed that the energy cost used is $6.21 \pm 1.01 \mathrm{kcal} \cdot \mathrm{min}^{-1}$, while the pulse rate is $129.5 \pm 18.4$ per minute, which is about $60 \%$ of the maximum pulse rate. Similarly, studies conducted by Phillips \& Ziuraitis [27] on energy costs for traditional resistance training where the energy cost recorded is $4.52 \pm 1.3 \mathrm{kcal} \cdot \mathrm{min}^{-1}$ with MET $3.9 \pm 0.4$ for men and $4.2 \pm 0.6$ for women. While Alcaraz, Sánchez-Lorente \& Blazevich [28] study of cardiovascular responses to two different training protocols, i.e. circuit resistance training and traditional strength training, found that the pulse rate for both exercises reached $71 \%$ intensity of maximum pulse rate for exercise circuit while $62 \%$ for traditional strength training.

\section{CONCLUSIONS}

Using rope and tire as tools for resistance training would give many advantages for the functionality development among the individuals. 


\section{ACKNOWLEDGEMENTS}

List here those individuals who provided help during the research (e.g., providing language help, writing assistance or proof reading the article, etc.).

\section{REFERENCES}

[1]. Boyle, M., Functional training for sports2004: Human Kinetics.

[2] Liebenson, C., Functional training handbook2014: Lippincott Williams \& Wilkins.

[3] Cosio-Lima, L.M., et al., Effects of physioball and conventional floor exercises on early phase adaptations in back and abdominal core stability and balance in women. The Journal of Strength \& Conditioning Research, 2003. 17(4): p. 721-725.

[4] Fleck, S.J. and W. Kraemer, Designing Resistance Training Programs, 4E2014: Human Kinetics.

[5] Nadzalan, A.M., et al., The effects of step versus jump forward lunge exercise training on muscle architecture among recreational badminton players. World Applied Sciences Journal, 2017. 35(8): p. 1581-1587.

[6] Nadzalan, A.M., et al., Relationship between lower body muscle architecture and lunges performance. Journal of Sports Science and Physical Education, Malaysia, 2016. 5(2): p. 15-23.

[7] Silva-Grigoletto, M.E.D., C.J. Brito, and J.R. Heredia, Functional training: functional for what and for whom? Revista Brasileira de Cineantropometria \& Desempenho Humano, 2014. 16(6): p. 714-719.

[8] McGill, S., Ultimate back fitness and performance2009: Backfitpro Incorporated.

[9] Ratamess, N.A., ACSM's foundations of strength training and conditioning2012: Wolters Kluwer Health/Lippincott Williams \& Wilkins.

[10]Bennett, S., Using "strongman" exercises in training. Strength \& Conditioning Journal, 2008. 30(3): p. 42-43.

[11]Hedrick, A., Using uncommon implements in the training programs of athletes. Strength \& Conditioning Journal, 2003. 25(4): p. 18-22.

[12]Bullock, J.B. and D.M. Aipa, Coaching considerations for the tire flip. Strength \& Conditioning Journal, 2010. 32(5): p. 75-78. 
[13]Keogh, J.W., et al., A brief description of the biomechanics and physiology of a strongman event: The tire flip. The Journal of Strength \& Conditioning Research, 2010. 24(5): p. 1223-1228.

[14]Cissik, J.M., Strength training tools for the track and field coach: A brief review. Track and Cross Country Journal, 2012. 1(4): p. 4-8.

[15]Climstein, M. and J. Walsh, Research Review: Exercise can be so demanding. Network, 2014: p. 49.

[16]Fountaine, C.J. and B.J. Schmidt, Metabolic cost of rope training. The Journal of Strength \& Conditioning Research, 2015. 29(4): p. 889-893.

[17]Nieman, D.C., et al., Validation of Cosmed's FitMate ${ }^{\mathrm{TM}}$ in measuring oxygen consumption and estimating resting metabolic rate. Research in Sports Medicine, 2006. 14(2): p. 89-96.

[18]Garber, C.E., et al., American College of Sports Medicine position stand. Quantity and quality of exercise for developing and maintaining cardiorespiratory, musculoskeletal, and neuromotor fitness in apparently healthy adults: guidance for prescribing exercise. Medicine and Science in Sports and Exercise, 2011. 43(7): p. 1334-1359.

[19]Ainsworth, B.E., et al., 2011 Compendium of Physical Activities: a second update of codes and MET values. Medicine and Science in Sports and Exercise, 2011. 43(8): p. $1575-1581$.

[20]Welk, G., Physical activity assessments for health-related research2002: Human Kinetics. [21]Plowman, S.A. and D.L. Smith, Exercise physiology for health fitness and performance2013: Lippincott Williams \& Wilkins.

[22]Jette, M., K. Sidney, and G. Blümchen, Metabolic equivalents (METS) in exercise testing, exercise prescription, and evaluation of functional capacity. Clinical Cardiology, 1990. 13(8): p. 555-565.

[23]Raven, P., et al., Exercise Physiology2012: Nelson Education.

[24]Ainsworth, B.E., et al., Compendium of physical activities: an update of activity codes and MET intensities. Medicine and Science in Sports and Exercise, 2000. 32(9; SUPP/1): p. S498-S504. 
[25]Scott, C.B., A. Croteau, and T. Ravlo, Energy expenditure before, during, and after the bench press. The Journal of Strength \& Conditioning Research, 2009. 23(2): p. 611-618.

[26]Beckham, S. and C.P. Earnest, Metabolic cost of free weight circuit weight training. Journal of Sports Medicine and Physical Fitness, 2000. 40(2): p. 118.

[27]Phillips, W.T. and J.R. Ziuraitis, Energy cost of the ACSM single-set resistance training protocol. Journal of Strength and Conditioning Research, 2003. 17(2): p. 350-355.

[28]Alcaraz, P.E., J. Sánchez-Lorente, and A.J. Blazevich, Physical performance and cardiovascular responses to an acute bout of heavy resistance circuit training versus traditional strength training. The Journal of Strength \& Conditioning Research, 2008. 22(3): p. $667-671$.

\section{How to cite this article:}

Iskandar M. M, Mohamad N. I, Othman S. and Nadzalan A. M. Metabolic Cost during Tyre and Rope Functional Training. J. Fundam. Appl. Sci., 2017, 9(6S), 1051-1062. 